\title{
Beim primären Hochrisiko-Melanom: Länger Interferon, länger leben?
}

\section{Wenn die Lymphknoten noch nicht befallen sind, profitieren Patienten mit malignem Melanom von einer Therapie mit Interferon $\alpha$ in niedriger Dosierung (LDI). Üblicherweise dauert diese Behandlung 18 Monate. Aber ist das auch genug, insbesondere für Patienten mit intermediärem oder hohem Risiko?}

$\mathrm{N}$ iedrig dosiertes Interferon $\alpha$ (IFN $\alpha)$ ermöglicht Melanom-Patienten verschiedenen klinischen Studien zufolge ein längeres krankheitsfreies Überleben, sofern noch kein klinisch erkennbarer Lymphknotenbefall vorliegt. Allerdings

\section{Nicht signifikante Überlebensraten}

\begin{tabular}{lll} 
Überleben: & Arm A & Arm B \\
\hline Rezidivfrei & $75,6 \%$ & $72,6 \%$ \\
\hline Fernmetastasenfrei & $81,9 \%$ & $79,7 \%$ \\
\hline Gesamtüberleben & $85,9 \%$ & $84,9 \%$
\end{tabular}

ist die optimale Länge der Behandlung weiter in der Diskussion. Möglicherweise könnte die Verlängerung der Interferon-Therapie von eineinhalb auf fünf Jahre von klinischem Nutzen für Melanom-Patienten mit mittlerem oder hohem Risiko sein. Eine multizentrische deutsch-österreichische Studie ist dieser Frage nachgegangen.

An der prospektiven, randomisierten und kontrollierten Studie nahmen 850 Patienten mit einem resezierten kutanen Melanom von mindestens 1,5 mm Dicke teil. Bei den Patienten hatte sich klinisch kein Lymphknoten-Befall gezeigt, eine Biopsie des Sentinel-Lymphknotens war in den meisten, aber nicht in allen Fällen durchgeführt worden. Die Patienten in Arm A erhielten 18 Monate lang dreimal wöchentlich drei Millionen Einheiten IFN $\alpha-2 a$, die in Arm B bekamen dieselbe Dosierung 60 Monate lang.

Nach einem medianen Follow-up von 4,3 Jahren konnten die Ergebnisse von 840 der 850 Patienten ausgewertet werden. Die Tumordicke und andere relevante prognostische Faktoren waren zwischen den beiden Armen ausgeglichen. Die Biopsie des Sentinel-Lymphknotens war bei 635 Patienten durchge- führt worden (75,6\%); in Arm A ergab sich dabei bei 18,0\% ein positives Ergebnis und in Arm B bei 17,5\%.

Statistisch signifikante Unterschiede zwischen den beiden Behandlungsarmen ergaben sich weder beim rezidivfreien Überleben (Arm A: 75,6\%; Arm B: $72,6 \% ; p=0,72$; Hazard Ratio 1,05; $95 \%$-KI $0,80-1,39)$ noch beim metastasenfreien Überleben (81,9\% / 79,7\%) oder beim Gesamtüberleben $(85,9 \%$ / $84,9 \%)$. Auch wenn nach median 4,3 Jahren Follow-up noch nicht einmal alle Patienten in Arm B ihre adjuvante Interferon-Behandlung beendet hatten, müsse man angesichts dieser Überlebensdaten konzedieren, so die Autoren, dass ein signifikanter Unterschied zwischen den Armen auch in Zukunft nicht zu erwarten sei.

\section{Nie wieder lachen?}

$U$ $m$ dies zu bestätigen, machte die Arbeitsgruppe im Abstand von acht Jahren bei 122 Frauen (Alter 10-72 Jahre, Hauttyp I-VI) standardisierte Fotos mit und ohne Lächeln und quantifizierte das Ausmaß der Gesichtsfalten mit Hilfe einer computerbasierten Bildauswertung. Zudem ermittelte sie zu Beginn Farbe, Hydratation, Sebum und $\mathrm{pH}$ Wert der Haut und zu beiden Zeitpunkten anhand von strukturierten Interviews Informationen zu Demografie und Lebensstil.

Bei allen Frauen zeichnete sich das individuelle Muster der persistierenden Falten auf den Fotos ohne Lachen nach acht Jahren bereits auf den Fotos mit Lachen zu Studienbeginn ab. Eine tro-

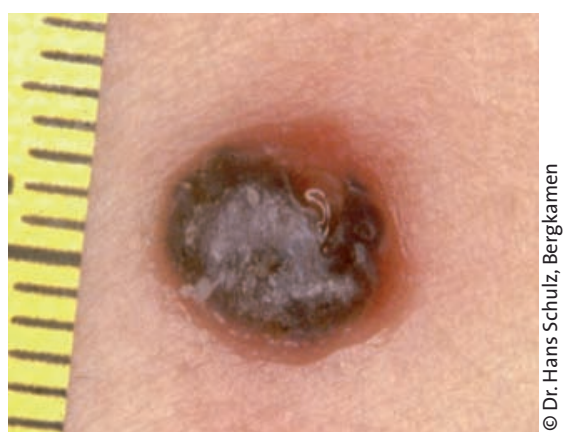

Eine verlängerte Interferon-Therapie ist hier fraglich: Knotiges Melanom am Oberarm bei einem 63jährigen Mann.

Fazit: Bei Patienten mit einem primären Melanom von mittlerem oder hohem Risiko ergibt sich durch eine Verlängerung der Therapie mit niedrigdosiertem Interferon von 18 auf 60 Monate kein klinischer Vorteil. Die Behandlung sollte also nicht länger als die bisher üblichen 18 Monate durchgeführt werden. be

Hauschild $A$ et al. Efficacy of low-dose interferon $\alpha 2 a 18$ versus 60 months of treatment in patients with primary melanoma of $\geq 1,5 \mathrm{~mm}$ tumor thickness: Results of a randomized phase III DeCOG trial. J Clin Oncol 2010; 28: 841-6

Dass Sonnenexposition eine wichtige Rolle bei der Faltenbildung spielt, ist allgemein anerkannt. Nach Ansicht von Forschern aus Japan und den USA wird jedoch der Einfluss der Mimik unterschätzt.

ckenere, hellere Haut mit niedrigerem $\mathrm{pH}-$ Wert und ein mittleres Alter (in den 40ern) zu Beginn sowie der Eintritt der Menopause in den acht Studienjahren waren mit einer stärkeren Bildung von persistierenden Falten assoziiert.

Fazit: Wiederholt auftretende Gesichtfalten, die durch die Mimik verursacht werden, können demnach zu persistierenden Falten führen. Mehrere intrinsische und extrinsische Faktoren beeinflussen diesen Prozess.

pe

Hillebrand GG et al. New wrinkles on wrinkling: an 8-year longitudinal study on the progression of expression lines into persistent wrinkles. Br J Dermatol 2010;162:1233-41 